\title{
THE ORDERABILITY AND SUBORDERABILITY OF METRIZABLE SPACES \\ BY
}

\author{
S. PURISCH
}

\begin{abstract}
A space is defined to be suborderable if it is embeddable in a (totally) orderable space. It is shown that a metrizable space $X$ is suborderable iff (1) each component of $X$ is orderable, (2) the set of cut points of each component of $X$ is open, and (3) each closed subset of $X$ which is a union of components has a base of clopen neighborhoods. Note that condition (1) and hence this result is topological since there are many good topological characterizations of connected orderable spaces.

In a space $X$ let $Q$ denote the union of all nondegenerate components each of whose noncut points has no compact neighborhood. It is also shown that a metrizable space $X$ is orderable iff (1) $X$ is suborderable, (2) $X-Q$ is not a proper compact open subset of $X$, and (3) if $W$ is a neighborhood of $p \in X$ and $K$ is the component in $X$ containing $p$ such that $(W-K)-Q$ has compact closure and $\{p\}$ is the intersection of the closures of $(W-K)$ $-Q$ and $(W-K) \cap Q$, then $K$ is a singleton. Corollaries are given; every condition in each of these corollaries is concisely stated and sufficient for a space to be orderable when it is metrizable and suborderable.

Both of these results are extended to a class properly containing the metrizable spaces.
\end{abstract}

1. Introduction. A space is suborderable if it can be embedded in a (totally) orderable space. Suborderability is also called generalized orderability (see [L]). Čech [C, pp. 285-286] proved that a space is suborderable iff there exists a total order on it such that (1) the original topology is finer than the open interval topology, and (2) each of its points has a local base consisting of (possibly degenerate) intervals. Clearly, intervals can be changed to convex sets here.

The purpose of this paper is to give easily applicable topological characterizations of orderable and suborderable metrizable spaces, thus answering a question of Herrlich $\left[\mathbf{H}_{5}\right]$.

There have been topological characterizations of suborderability and order-

Presented to the Society, January 28, 1973 under the title On the suborderability of metric spaces and Stone-Cech compactifications; received by the editors April 25, 1975 and, in revised form, September 9, 1975.

AMS (MOS) subject classifications (1970). Primary 06A05, 54F05, 54E35; Secondary 54F45, S4D35.

Key words and phrases. Totally orderable space, suborderable space, metrizable space, convex metric, cut point, clopen neighborhood base, $\varepsilon$-decomposition, large inductive dimension zero, neighbors, ordered compactification. 
ability for many classes of spaces. The most important ones have been obtained for orderable connected spaces in 1941 by Eilenberg [E], for orderable totally disconnected metrizable spaces and suborderable separable metrizable spaces in 1962 by Herrlich [ $\left.\mathrm{H}_{1}\right]$, for orderable separable metrizable spaces that are subordered in 1965 by M. E. Rudin [Ru], and for arbitrary suborderable and orderable spaces in 1973 by van Dalen and Wattel [D-W].

One might believe that an orderable metrizable space is locally separable. If this were true a topological characierization of an orderable metrizable space would be a simple corollary of results by Herrlich $\left[\mathrm{H}_{4}\right]$ and $\mathrm{M}$. E. Rudin [Ru]. However the cartesian product of an uncountable discrete space with itself a countable number of times is a counterexample.

2. Basic definitions and preliminary results. In this paper $\mathbf{N}$ will be the positive integers, and $\mathbf{R} \mathbf{v}$ ill be the real line each with its usual order.

Let $(X,<)$ be a totally ordered set. For $a \in X$ let $(-\infty, a)=\{x \in X \mid x<$ a) and $(a, \infty)=\{x \in X \mid a<x\}$.

Let $<$ be a total order on a topological space $X$. The pair $(X,<)$ is called an ordered space and $<$ is called an admissible order on $X$ if the open interval topology coincides with the original topology. The pair $(X,<)$ is called a subordered space and $<$ is called an admissible suborder on $X$ if $(X,<)$ can be embedded by an order-preserving map into an ordered space. Hence, $(X,<)$ is subordered and $<$ is an admissible suborder on $X$ iff $(X,<)$ satisfies conditions (1) and (2) of the result of Čech given in the introduction of this paper.

Where it will cause no confusion, we shall omit mention of $<$ and simply write "let $X$ be an ordered set," "let $X$ be an ordered space," or "let $X$ be a subordered space."

A compact ordered space $S$ is said to be an ordered compactification of a subordered space $X$ if $X$ can be densely embedded by an order preserving map into $S$.

If $X$ is subordered and $y \in X$, then $y$ is called a left limit point of $X$ if it is in the closure of $(-\infty, y)$, and $y$ is called a right limit point of $X$ if it is in the closure of $(y, \infty)$

An element $p$ of a connected space $C$ is called a cut point of $C$ if $C-\{p\}$ is not connected, and $p$ is called a noncut point of $C$ if $C-\{p\}$ is connected.

In Proposition 2.1 properties are given of condition (3) of Theorem 3.4, the main suborderability result. These properties will often be used in §3. Propositions 2.2 and 2.3 are also needed for the next section.

A clopen neighborhood base $\mathfrak{B}$ of a subset $A$ of a space $X$ is a collection of clopen subsets of $X$ containing $A$ such that for each open subset of $X$ containing $A$ there is a member of $\mathscr{B}$ contained in the open set. 
The proof of the following proposition is straightforward and, hence, omitted.

2.1. Proposition. Let $X$ be a $T_{2}$ space each of whose components has a clopen neighborhood base and a finite number (perhaps zero) of boundary points. Then each of the following properties holds:

(1) Every point of $X$ has a base of open neighborhoods each member of which has its boundary contained in the point's component.

(2) Every point of $X$ has a base of open neighborhoods each member of which is a union of components plus a piece of the point's component.

(3) If a net $\left\{x_{\alpha}\right\}_{\alpha \in Q}$ in the complement of a component converges to a point $p$ in the given component, and if $x_{\alpha}^{\prime}$ is a point in the component of $x_{\alpha}$ for each $\alpha \in \mathbb{Q}$, then $\left\{x_{\alpha}^{\prime}\right\}_{\alpha \in \mathbb{Q}}$ converges to $p$.

Recall that a decomposition of a set is a cover of the set by nonempty pairwise disjoint subsets. An $\varepsilon$-decomposition $\left[\mathbf{H}_{2}\right]$ of a metric space for $\varepsilon>0$ is a decomposition of the space each member of which is open and has diameter not greater than $\varepsilon$. A metric space is decomposable $\left[\mathbf{H}_{2}\right]$ if for every $\varepsilon>0$ the space has an $\varepsilon$-decomposition. Recall also that Ind of a space $X$ equals zero if each closed subset of $X$ has a clopen neighborhood base.

2.2. Proposition $\left[\mathrm{H}_{2}\right]$. For a totally disconnected metric space $\mathrm{X}$ the following are equivalent:

(1) $X$ is orderable.

(2) $X$ is decomposable.

(3) Ind $X=0$.

The proof in [ $\mathbf{H}_{2}$, Lemma 1], that the Ind of each totally disconnected orderable space equals zero is also a proof of the following proposition.

2.3. Proposition. The Ind of a totally disconnected suborderable space equals zero.

Propositions 2.4 and 2.5 will be used in Lemma 4.4, which is the key lemma in proving the orderability theorem given in $\$ 4$.

A metric $\partial$ on an ordered set $X$ is called a convex metric if, for every $a<b<c<d$ in $X, \partial(b, c)<\partial(a, d)$.

2.4. Proposition. A metric $\partial$ on an ordered set $X$ is convex iff for every $u, v$, $w \in X$ such that $u<v<w, \max \{\partial(u, v), \partial(v, w)\}<\partial(u, w)$.

A metric on a space is called an admissible metric if the metric topology coincides with the original topology.

If $X$ is a metrizable subordered space, it has an admissible metric $\rho$ bounded by 1 . Then there is an admissible convex metric $\partial$ on $X$ bounded by 1 as follows: for $x, y \in X$ and $x<y$ let 


$$
\partial(x, y)=\partial(y, x)=\sup \left\{\rho\left(z, z^{\prime}\right) \mid x<z<z^{\prime}<y, \text { where } z, z^{\prime} \in X\right\} .
$$

So we obtain the next result.

2.5. Proposition. If $X$ is a metrizable subordered space, then there is an admissible convex metric on $X$ bounded by 1 .

3. The suborderability of metrizable spaces. Let $X$ be a space whose components each have at most two boundary points. Choose $U \subseteq X$ as follows: (1) If $K$ is a singleton or open component of $X$, then choose one point from $K$ to be in $U$. (2) If $K$ is a nondegenerate nonopen component of $X$, then choose two points from $K$, including its boundary points, to be in $U$.

Note that if $X$ satisfies conditions (1) and (2) in each of 3.1-3.4, then each component of $X$ has at most two boundary points. In addition, if $X$ satisfies condition (3) in each of 3.1-3.4, then any two subsets of $X$ chosen to satisfy the requirements in the definition of $U$ are horneomorphic. Hence $U$ is not as arbitrary as one might first believe.

Suppose $U$ has been chosen as a subspace of a space $X$. Two distinct points in $U$ are called neighbors if they belong to the same component in $X$.

It should be pointed out that condition (1) in the following lemma is topological since there are many good topological characterizations of orderable connected spaces. For example see [E], [ $\left.\mathbf{H}_{3}\right]$, [Kok], and [Kow, p. 109].

The proof of the following lemma is tedious but not difficult, so it will not be given here. However it should be pointed out that in the sufficiency proof one would find it helpful to use properties (2) and (3) of Proposition 2.1.

3.1. LEMMA. $A$ space $X$ is suborderable iff:

(1) Each component of $X$ is orderable.

(2) The set of cut points of each component of $X$ is open.

(3) Each component of $X$ has a clopen neighborhood base.

(4) There exists an admissible subordering $\leqslant$ on $U$ such that there are no points of $U$ between neighbors with respect to $<$.

3.2. LemMA. $A$ metric space $X$ is suborderable iff:

(1) Each component of $X$ is orderable.

(2) The set of cut points of each component of $X$ is open.

(3) Each component of $X$ has a clopen neighborhood base.

(4) For every $\varepsilon>0$ there is an $\varepsilon$-decomposition of $U$ each member of which contains the neighbors of each of its points with possibly one exception.

Proof. If $X$ is suborderable, then conditions (1), (2), and (3) are true. It will now be shown that condition (4) is true. Let $\varepsilon>0$. Clearly, $U$ is totally disconnected. As a corollary of Propositions 2.2 and 2.3 we have that a totally disconnected metric space is suborderable iff it is decomposable. Hence, $U$ is decomposable since it is a suborderable metric space. So let $W$ be an 
$\varepsilon$-decomposition of $U$, and let $<$ be an admissible subordering on $X$. Each maximal convex subset of every member of $\mathscr{W}$ is clopen in $U$ and contains the neighbors of each of its points with possibly two exceptions. It is easy to show that since $X$ is suborderable, $X$ satisfies property (1) of Proposition 2.1. Using this fact we obtain a decomposition of cardinality at most two of each of these maximal convex subsets such that each member of every decomposition is open in $U$ and contains the neighbors of each of its puints with possibly one exception. Hence, if the neighbors of two of the points in some maximal convex subset of some member of $\mathcal{W}$ are not in this subset, then these two points are in different members of the decomposition of this subset. Then the union of the decompositions of all the maximal convex subsets of each of the members of $\mathcal{W}$ is an $\varepsilon$-decomposition of $U$ each member of which contains the neighbors of each of its points with possibly one exception.

Conversely, let conditions (1)-(4) hold. If we can show that condition (4) of Lemma 3.1 holds, then we will have proven $X$ is suborderable. (The following proof is similar to, but contains essential nontrivial deviations from, a proof that a decomposable space is orderable in [ $\mathbf{H}_{2}$, Lemma 3].) Let $\varepsilon>0$ and let W be an $\varepsilon$-decomposition of $U$ each member of which contains the neighbors of each of its points with possibly one exception. An order $<$ on $W$ is called a W ordering if two distinct members of 0 which contain neighbors have no other member of $\mathscr{W}$ between them with respect to $\leqslant$. Clearly there exists a थ ordering.

Now let $<$ be a $W$ ordering. A function

$$
f=(g, h): \text { W } \rightarrow(U \times\{\varnothing\}) \cup(\{\varnothing\} \times U) \cup(\{\varnothing\} \times\{\varnothing\})
$$

is called a $\mho-<$ map if: $(1)(\varnothing, \varnothing)$ is the image of every member of $\mathscr{W}$ which contains the neighbors of each of its points, and (2) if $W$ and $W^{\prime}$ are distinct members of W which contain neighbors $y$ and $y^{\prime}$, respectively, such that $W<W^{\prime}$, then $f(W)=(\varnothing, y)$ and $f\left(W^{\prime}\right)=\left(y^{\prime}, \varnothing\right)$.

Note that since each member of $Q 5$ contains the neighbors of each of its points with possibly one exception, $f$ is unique and well defined.

We will construct an order on $U$ by induction satisfying condition (4) of Lemma 3.1 as follows. Let $\mathcal{W}_{1}$ be a $2^{-1}$ decomposition of $U$ each member of which contains the neighbors of each of its points with possibly one exception, let $\leqslant_{1}$ be a $\mathcal{W}_{1}$ ordering, and let $f_{1}=\left(g_{1}, h_{1}\right)$ be a $\mathscr{W}-\leqslant_{1}$ map.

Assume we have defined a $2^{-n}$ decomposition $\mathscr{W}_{n}$ of $U$ each member of which contains the neighbors of each of its points with possibly one exception, a $\mho_{n}$ ordering $\leqslant_{n}$, and a $\mho_{n}-\leqslant_{n} \operatorname{map} f_{n}=\left(g_{n}, h_{n}\right)$. We will show first that for each $W \in \mathcal{W}_{n}$ there is a $2^{-n-1}$ decomposition $\mathcal{W}(W)$ of $W$ each member of which contains the neighbors of each of its points with possibly one exception. By construction each $W \in \mathcal{W}_{n}$ contains the neighbors of each of its points with possibly one exception. So if there is an element $x$ of the 
open set $W$ whose neighbor is not in $W$, then there exists a natural number $m>n$ such that $2^{-m}<\partial(x, U-W)$, where $\partial$ is the metric on $X$. Let $\mathcal{G}$ be a $2^{-m}$ decomposition of $U$ each member of which contains the neighbors of each of its points with possibly one exception, and let $G_{x}$ be the element of $\mathcal{G}$ containing $x$. Then $G_{x} \subseteq W$, and $G_{x}$ contains the neighbors of each of its points with the exception of $x$ 's neighbor. If $W$ contains the neighbors of each of its points let $\mathcal{G}$ be a $2^{-n-1}$ decomposition of $U$. In either case $\mho(W)=$ $\{W \cap G \mid W \cap G \neq \varnothing$ and $G \in \mathcal{G}\}$ is a $2^{-n-1}$ decomposition of $W$ each member of which contains the neighbors of each of its points with possibly one exception.

It is easy to show that there exists an order $<w_{n+1}$ on $\mathscr{W}(W)$ such that (1) two distinct members of $W(W)$ which contain neighbors have no other members of $Q(W)$ between them with respect to $<w_{n+1}$, and (2) if $g_{n}(W) \neq$ $\varnothing$, then $g_{n}(W)$ is in the first element of $\left(\mathcal{W}(W),<w_{n+1}\right)$, and if $h_{n}(W) \neq \varnothing$, then $h_{n}(W)$ is in the last element of $\left(W(W),<w_{n+1}\right)$. Call such an order a W $(W)$ ordering.

Clearly, $\mathcal{W}_{n+1}=U\left\{W(W) \mid W \in \mathcal{W}_{n}\right\}$ is a $2^{-n-1}$ decomposition of $U$ each member of which contains the neighbors of each of its points with possibly one exception.

Define an order $<_{n+1}$ on $\mathcal{W}_{n+1}$ as follows. For each $A, B \in \mathcal{W}_{n+1}$ and each $V, W \in W_{n}$ such that $A \in \mathcal{W}(V)$ and $B \in \mathcal{W}(W)$, define $A<_{n+1} B$ iff $A<w_{n+1} B$ whenever $V=W$, and $A<_{n+1} B$ iff $V<_{n} W$ whenever $V \neq W$. Then it is routine to show $<_{n+1}$ is a $\mho_{n+1}$ ordering.

Let $f_{n+1}=\left(g_{n+1}, h_{n+1}\right)$ be the unique $\mho_{n+1}-<_{n+1}$ map.

Define an order $<$ on $U$ as follows. For $a, b \in U$ define $a<b$ iff $a=b$ or there exists an $n \in \mathbf{N}$ and two distinct members $V$ and $W$ of $2 \int_{n}$ such that $a \in V$, and $b \in W$ and $V<_{n} W$. It is now not difficult to finish the proof by showing $<$ is well defined and it is an admissible suborder on $U$ such that there are no points of $U$ between neighbors with respect to $<$.

Roy's space $\Delta[$ Ro] shows the insufficiency of conditions (1), (2), and (3) in Theorem 3.3 since $\Delta$ is a totally disconnected 0 -dimensional metric space but Ind $\Delta \neq 0$.

3.3. Theorem. A metrizable space $X$ is suborderable iff:

(1) Each component of $X$ is orderable.

(2) The set of cut points of each component of $X$ is open.

(3) Each component of $X$ has a clopen neighborhood base.

(4) The Ind of every totally disconnected subspace of $X$ equals zero.

Proof. If $X$ is suborderable, then conditions (1), (2), and (3) are true. As a corollary of Propositions 2.2 and 2.3 we have a totally disconnected metrizable space $Y$ is suborderable iff Ind $Y=0$. Hence condition (4) is true because every subspace of $X$ is metrizable. 
Conversely, let conditions (1)-(4) hold. For the rest of this proof open and closed sets and closures are relative to $U$ unless stated otherwise. To prove that $X$ is suborderable it is sufficient to show that condition (4) of Lemma 3.2 holds. Let $\varepsilon>0$ and let $\partial$ be an admissible metric on $U$. In Proposition 2.2 it is stated that the Ind of a totally disconnected metric space equals zero iff it is decomposable. Hence, by condition (4) of this theorem there exists an $\varepsilon$-decomposition $\mho$ of $U$ with respect to $\partial$. Let $W \in W$. Let $B_{W}$ be the set of points of $W$ whose neighbors are in $U-W . B_{W}$ has no accumulation points because if there was a net in $B_{W}$ converging to $x \in U$, then the neighbors of the points of this net must converge to $x$ by property (3) of Proposition 2.1. But this is impossible since $W$ is clopen.

Since $B_{W}$ contains no accumulation points, it is hereditarily closed. So for each $x \in B_{W}, \partial\left(x, B_{W}-\{x\}\right)=\delta_{x}>0$. Then since $W$ is open there exists an open neighborhood $O_{x}$ of $x$ contained in $W$ of diameter less than $\delta_{x} / 2$. Hence, $\left\{O_{x} \mid x \in B_{W}\right\}$ is a pairwise disjoint collection of nonempty open sets, $\cup\left\{O_{x} \mid x \in B_{W}\right\}$ is an open neighborhood of $B_{W}$ contained in $W$, and $O_{x} \cap B_{W}=\{x\}$ for each $x \in B_{W}$. By condition (4) of this theorem Ind $U$ $=0$ since $U$ is totally disconnected. So there exists a clopen neighborhood $F$ of $B_{W}$ contained in $\cup_{x \in B_{W}} O_{x}$ since $B_{W}$ is a closed subset of $U$. Thus, for each $x \in B_{W}, O_{x} \cap F$ is open, $\left\{O_{x} \cap F \mid x \in B\right\}$ is a pairwise disjoint collection of nonempty open sets, and $\cup_{x \in B_{W}}\left(O_{x} \cap F\right)=F$ is clopen. By property (1) of Proposition 2.1, each $x \in B_{W}$ has a base of neighborhoods in $X$ each member of which has its boundary in $x$ 's component and does not contain $x$ 's neighbor in its closure. Then there exists such a neighborhood $O_{x}^{\prime \prime}$ of $x$ such that $O_{x}^{\prime}=O_{x}^{\prime \prime} \cap U \subseteq O_{x} \cap F$. Since $O_{x}^{\prime \prime}$ has its boundary in $x$ 's component $K_{x}, O_{x}^{\prime \prime}-K_{x}$ is a union of components. Hence, $O_{x}^{\prime}$ contains the neighbors of each of its points with the exception of $x$ 's neighbor, and $O_{x}^{\prime}$ has no boundary in $U$ because $O_{x}^{\prime \prime}$ does not contain $x$ 's neighbor in its closure. So $O_{x}^{\prime}$ is clopen in $U$.

Let us show that $\cup_{x \in B_{W}} O_{x}^{\prime}$ is a clopen neighborhood of $B_{W}$ contained in $W$. Since each $O_{x}^{\prime}$ is an open neighborhood of $x$ contained in $W, \cup_{x \in B_{W}} O_{x}^{\prime}$ is an open neighborhood of $B_{W}$ contained in $W$. So let $y \in \bar{U}_{x \in B_{W}} O_{x}^{\prime}$. Since $\overline{\bigcup_{x \in B_{W}} O_{x}^{\prime}} \subseteq \overline{U_{x \in B_{W}}\left(O_{x} \cap F\right)}=\bar{F}=F, y \in F=\cup_{x \in B_{W}}\left(O_{x} \cap F\right)$. Hence, there exists an $x_{0} \in B_{W}$ such that $y \in O_{x_{0}} \cap F$. Since $\left\{O_{x} \cap F \mid x \in B_{W}\right\}$ is pairwise disjoint and, for every $x \in B_{W}, O_{x}^{\prime} \subseteq O_{x} \cap F, O_{x_{0}} \cap F$ and $\bigcup_{x \neq x_{0}} O_{x}^{\prime}$ are disjoint open sets. Therefore, $y \notin \overline{\bigcup_{x \neq x_{0}} O_{x}^{\prime}}$. So $y \in \overline{O_{x_{0}}^{\prime}}=O_{x_{0}}^{\prime}$. Hence, $\cup_{x \in B_{W}} O_{x}^{\prime}$ is closed. Thus, $\cup_{x \in B_{W}} O_{x}^{\prime}$ is a clopen neighborhood of $B_{W}$ contained in $W$.

Since $W$ is open and each $O_{x}^{\prime}$ is clopen and contains the neighbors of each of its points with the exception of $x$ 's neighbor, $W-\cup_{x \in B_{W}} O_{x}^{\prime}$ is an open subset of $U$ which contains the neighbors of each of its points. Let $\mathscr{Q}(W)=$ $\left\{O_{x}^{\prime} \mid x \in B_{W}\right\} \cup\left\{W-\cup_{x \in B_{W}} O_{x}^{\prime}\right\}$. Then $\mathscr{W}(W)$ is an $\varepsilon$-decomposition of 
$W$ each member of which contains the neighbors of each of its points with possibly one exception. Hence, $\cup\{\mathcal{W}(W) \mid W \in \mathscr{V}\}$ is an $\varepsilon$-decomposition of $U$ each member of which contains the neighbors of each of its points with possibly one exception.

Therefore, condition (4) of Lemma 3.2 is satisfied. So $X$ is suborderable.

Note that in Theorem 3.3 we could have replaced condition (4) by the condition that Ind $U$ equals zero or equivalently $U$ is decomposable since this is the only use of condition (4) in this theorem.

3.4. TheOREM. A metrizable space $X$ is suborderable iff:

(1) Each component of $X$ is orderable.

(2) The set of cut points of each compcnent of $X$ is open.

(3) Each closed subset of $X$ which is a union of components has a clopen neighborhood base.

Proof. Necessity is clear. So let conditions (1), (2), and (3) hold. By Theorem 3.3 and the note immediately after the proof of Theorem 3.3, it is sufficient in proving this half of this theorem to show that Ind $U=0$.

Before proving that Ind $U=0$, two more preliminary results must be shown: that $U$ is closed and that every closed subset of $U$ which contains the neighbors of all its points has a $U$-clopen neighborhood base. Let $y$ be an accumulation point of $U$. Since there are at most two points in the intersection of $U$ and any component of $X, y$ is a boundary point of its component. Therefore, by the definition of $U, y \in U$. Hence $U$ is closed.

Now let $C$ be a closed subset of $U$ which contains the neighbors of all its points, and let $O$ be a $U$-open neighborhood of $C$. Since $U$ is closed in $X, C$ is closed in $X$. For each $x \in C$ let $K_{x}$ be the component containing $x$ in $X$. Then $\left(\cup_{x \in C} K_{x}\right) \cap U=C$. By property (3) of Proposition 2.1 every boundary point of $\cup_{x \in C} K_{x}$ is a boundary point of the closed set $C$. Hence, $\cup_{x \in C} K_{x}$ is closed because it contains $C$. Now there is an open set $O^{\prime}$ of $X$ such that $O^{\prime} \cap U=O$. Then $O^{\prime} \cup\left(\cup_{x \in C} K_{x}\right)$ is open in $X$ since every boundary point of $K_{x}$ for each $x \in C$ is a point of $U$ and, hence, is in the closed set $C$ which in turn is contained in $O^{\prime}$. Since every closed subset of $X$ which is a union of components has a clopen neighborhood base, there is a clopen neighborhood $V$ of $\cup_{x \in C} K_{x}$ contained in $O^{\prime} \cup\left(\cup_{x \in C} K_{x}\right)$. Hence, $V \cap U$ is a clopen neighborhood of $C$ relative to $U$ which is contained in $U \cap\left(O^{\prime} \cup\left(\cup_{x \in C} K_{x}\right)\right)=O$. Therefore, $C$ has a clopen neighborhood base in $U$.

We are now ready to show that Ind $U$ equals zero. For the remainder of this proof let open and closed sets and closures be taken relative to $U$. Let $C$ be a closed subset of $U, B_{C}$ the set of all points of $C$ whose neighbors are in $U-C, B_{C}^{\prime}$ the neighbors of the points of $B_{C}$, and let $O$ be an open 
neighborhood of $C$. We will show there is a clopen neighborhood of $C$ contained in $O$.

$B_{C}^{\prime}-O$ is closed. In fact, it has no accumulation points, since by property (3) of Proposition 2.1 each accumulation point of $B_{C}^{\prime}-O$ is also an accumulation point of $B_{C}$ and, hence, this point would be contained in the disjoint closed sets $C$ and $U-O$. Each accumulation point of $C \cup\left(B_{C}^{\prime} \cap\right.$ $O$ ) is either contained in $C$ or is an accumlation point of $B_{C}^{\prime} \cap O$. Again by property (3) of Proposition 2.1 each accumulation point of $B_{C}^{\prime} \cap O$ is an accumulation point of $B_{C}$, and, hence, is contained in $C$. So $C \cup\left(B_{C}^{\prime} \cap O\right)$ is also closed. Since $U$ is metric, it is normal. So there exists an open set $G$ such that $C \cup\left(B_{C}^{\prime} \cap O\right) \subseteq G \subseteq \bar{G} \subseteq O$. Hence, $G$ and $U-\bar{G}$ are disjoint open neighborhoods of $C \cup\left(B_{C}^{\prime} \cap O\right)$ and $B_{C}^{\prime}-O$ respectively. Since $C \cup\left(B_{C}^{\prime}\right.$ $\cap O)$ and $B_{C}^{\prime}-O$ are closed, $\left(C \cup\left(B_{C}^{\prime} \cap O\right)\right) \cup\left(B_{C}^{\prime}-O\right)=C \cup B_{C}^{\prime}$ is a closed set which contains the neighbors of all its points. Therefore, there exists a clopen neighborhood $P$ of $C \cup B_{C}^{\prime}$ contained in $G \cup(U-\bar{G})$. Finally, we need only show that $P \cap G$ is a clopen neighborhood of $C$ contained in $O$. Since $P$ and $G$ are open, $P \cap G$ is an open neighborhood of $C$ contained in $O$. Since $U-\bar{G}$ is open, $P \cap(U-\bar{G})$ is also open. Hence, $P \cap G$ is closed because $P$ is closed and $P-(P \cap(U-\bar{G}))=P \cap G$. Thus, $P \cap G$ is a clopen neighborhood of $C$ contained in $O$. So Ind $U=0$. Hence by Theorem 3.3 and the note immediately after Theorem 3.3, $X$ is suborderable.

In 3.2-3.4 the only use made of $X$ being metrizable is that $U$ should be metrizable. Hence, we have the next corollary.

3.5. Corollary. Let $X$ be a space each of whose components has at most two boundary points, and let $U$ be metrizable. Then $X$ is suborderable iff it satisfies conditions (1), (2), and (3) of Theorem 3.4.

4. The orderability of metrizable spaces. In a space $X$ let $Q$ denote the union of all nondegenerate components each of whose noncut points has no compact neighborhood.

We will now examine the types of components of a suborderable space $X$ contained in $Q$, and the types of components of $X$ contained in $X-Q$. Every singleton component of $X$ is contained in $X-Q$. A topologically open component is contained in $Q$ iff it has no endpoints. (Note that a component with no endpoints cannot be a singleton.) Any other type of component can either be contained in $Q$ or $X-Q$ depending on the local conditions of its endpoints. However, if $X$ is compact, $X=X-Q$, and if $X$ is locally compact, $Q$ is the union of all components of $X$ with no endpoints. Note that a nondegenerate compact component of a space $X$ may be contained in $Q$. For example, let $X$ be the subset of $\mathbf{R}$ which is the union of all the rational numbers less than 0 or greater than 1 with the interval $[0,1]$. Then $[0,1]$ is a 
nondegenerate compact component of $X$. Since there is no compact neighborhood of either of the noncut points, 0 and 1 of $[0,1],[0,1] \subseteq Q$.

Some examples will be given to motivate conditions (2) and (3) of 7 heorem 4.2 , the orderability theorem, because the statements of these conditions are awkward and, perhaps, confusing.

4.1. Examples. 1. The subspace $X=(1,2) \cup\{3\}$ of $\mathbf{R}$ is not orderable. Clearly $X$ satisfies condition (1) of Theorem 4.2. $Q=(1,2)$ is a propc: closed subset of $X$, and $X-Q=\{3\}$ is compact. Hence, $X$ does not satisfy condition (2) of Theorem 4.2. Since $X-Q$ is clopen and disjoint from $Q$, there can be no subset $W$ of $X$ such that $W-Q$ and $W \cap Q$ share a boundary point. Hence, $X$ satisfies condition (3) of Theorem 4.2, since the hypothesis of this condition cannot be satisfied.

2(a). Let $X=[-1,0] \cup\left(\cup_{n \in N} V_{n}\right)$, where for $n$ oad, $V_{n}=[1 /(2 n+1)$, $1 / 2 n]$, and for $n$ even, $V_{n}=(1 /(2 n+1), 1 / 2 n)$. Then $X$ is not orderable but satisfies condition (1) of Theorem 4.2. Since $Q=U\left\{V_{n} \mid n\right.$ is even $\}$ and the point 0 is an element of $\bar{Q}-Q, Q$ is not closed. Therefore, $X$ satisfies condition (2) of Theorem 4.2. Let $W=X-[-1,0]$. Then $W$ is a neighborhood of 0 relative to the complement of 0 's component $[-1,0]$ (see the definition just before Theorem 4.2) because $X-[-1,0]=W$ and $X$ is a neighborhood of 0 . Then $W-Q=\cup\left\{V_{n} \mid n\right.$ is odd $\}$, and $W \cap Q=$ $\cup\left\{V_{n} \mid n\right.$ is even $\}$. So $\overline{W-Q}$ is the compact set $\{0\} \cup\left(\cup\left\{V_{n} \mid n\right.\right.$ is odd $\left.\}\right)$, and $\{0\}$ is the boundary of both $W-Q$ and $W \cap Q$. However, 0 's component is the nondegenerate set $[-1,0]$. Thus $X$ does not satisfy condition (3) of Theorem 4.2.

2(b). Let $X$ be the space described in part (a). For an even natural number $n$ let $A_{n}=(-1 / 2 n,-1 /(2 n+1))$, and for an odd natural number $n$ let $B_{n}=[1 /(2 n+1), 1 / 2 n]$. Then $X^{\prime}=X-[-1,0)=\{0\} \cup\left(\cup\left\{V_{n} \mid n \in N\right\}\right)$ is orderable since it is homeomorphic to the ordered space $Y=\left(\cup\left\{A_{n} \mid n\right.\right.$ is even $\}) \cup\{0\} \cup\left(\cup\left\{B_{n} \mid n\right.\right.$ is odd $\left.\}\right)$ with the order induced by the natural ordering on $\mathbf{R}$. Note that $\{0\}$ is the boundary of both $Q=U\left\{V_{n} \mid n\right.$ even $\}$ and $X-Q$, where $Q$ is now defined in terms of $X^{\prime}$. In fact 0 is the only point $p \in X$ which has a neighborhood relative to the complement of $p$ 's component such that $p$ and this neighborhood satisfy the hypothesis of condition (3) of Theorem 4.2. However, 0 's component in $X^{\prime}$ is $\{0\}$ while in the nonorderable space $X, 0$ 's component is $[-1,0]$.

3. We will now define an orderable subspace $X$ of $\mathbf{R}$ which demonstrates that in the hypothesis of condition (3) of Theorem 4.2 it is necessary to stipulate that (using the notation of condition (3) of Theorem 4.2) $p$ is the only boundary point of $W-Q$ as well as the only boundary point of $W \cap Q$. Let $X=[-1,0] \cup\left(\cup\left\{W_{n} \mid n \in \mathbf{N}\right\}\right)$, where, for each $n \in \mathbf{N}$,

$$
W_{n}=\cup\left\{\left(\frac{1}{2^{n}}-\frac{1}{2 k 2^{n+1}}, \frac{1}{2^{n}}-\frac{1}{(2 k+1) 2^{n+1}}\right) \mid k \in \mathbf{N}\right\} \cup\left\{\frac{1}{2^{n}}\right\} \text {. }
$$




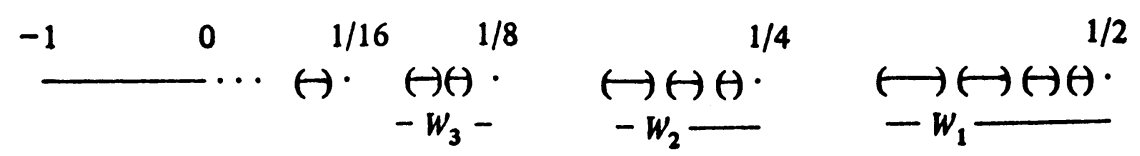

It should be clear how to construct an admissible order on $X . X-Q$ is the compact subset $[-1,0] \cup\left\{1 / 2^{n} \mid n \in \mathbf{N}\right\}$. Let $W=X-[-1,0]$. Then $W$ is a neighborhood of 0 relative to the complement of 0 's component $[-1,0]$, and $\overline{W-Q}$ is the compact set $\{0\} \cup\left\{1 / 2^{n} \mid n \in N\right\}$. However, $\{0\} \cup\left\{1 / 2^{n} \mid n \in\right.$ $\mathbf{N}\}$ is also the boundary of both $W-Q$ and $W \cap Q$, and 0 's component $[-1,0]$ is nondegenerate. In fact, for any neighborhood $V$ of 0 relative to the complement of 0 's component there exists an $m \in N$ such that $\{0\} \cup\left\{1 / 2^{n} \mid n\right.$ $>m\}$ is contained in the boundary of both $V-Q$ and $V \cap Q$.

Given a point $p$ of a space $X$, we define a subset $W$ of $X$ to be a neighborhood of $p$ relative to the complement of $p$ 's component $K$ if there exists a neighborhood $W^{\prime}$ of $p$ such that $W=W^{\prime}-K$.

4.2. THEOREM. A metrizable space $X$ is orderable iff:

(1) $X$ is suborderable.

(2) $X-Q$ is not a proper compact open subset of $X$.

(3) If $W$ is a neighborhood of $p \in X$ relative to the complement of $p$ 's component such that $W-Q$ has compact closure and $\{p\}$ is the intersection of the closures of $W-Q$ and $W \cap Q$, then $p$ 's component is a singleton.

In order to prove this theorem a special case of a result of M. E. Rudin [Ru] must be given, and two lemmas must be proven. The proof of Theorem 4.2 will be completed after Lemma 4.5.

In Lemma 4.3 we let $X$ be a first countable subspace of an ordered space $(T,<)$, and we let $\left(S,<^{\prime}\right)$ be an ordered compactification of $T$. The symbols $\mathscr{F}$ and $\mathcal{G}$ which appear in Lemma 4.3 and are defined in [Ru] have complicated definitions. So only the properties of $\mathscr{F}$ and $\mathcal{G}$ needed in subsequent proofs will be given. It can be easily verified by the reader that $\mathscr{F}$ and $\mathcal{S}$ do have the properties to be listed by looking at the places in [Ru] which will be indicated in this list. The properties of $\mathscr{F}$ and $\mathcal{G}$ that will be needed are:

(a) $\mathcal{G}$ is a collection of closed intervals of $\left(S,<^{\prime}\right)$ such that if $I, J \in \mathcal{G}$, then either $I=J, I \cap J=\varnothing$, or $I \cap J$ is a singleton. (See p. 388, II.A.(5) in [Ru].)

(b) $\mathcal{F}$ is a subset of $\mathcal{G}$ each member of which is nondegenerate and intersects $X$. (See p. 388, II.A.(6) in [Ru].)

(c) If $S \notin \mathcal{G}$, then no member of $\mathscr{F}$ contains $X$. (See p. 379, I.F.; p. 380, I.G.; p. 382, Lemma 10; pp. 387, 388, II, first paragraph; and p. 388, II.A.(5) and (6), all in [Ru].)

As an aid to the reader referring to [Ru] the symbols $T, X, \mathscr{F}, \mathcal{S}$, and $Q$ 
that appear here are the symbols $R, T, F, G$, and $Q$, respectively, in [Ru].

In Lemmas 4.3, 4.4, and 4.5 let closures be taken in $X$.

4.3. LEMMA [Ru]. Let $X$ be a first countable space embedded in an ordered space $(T, \leqslant)$, and let $\left(S,<^{\prime}\right)$ be an ordered compactification of $T$. Then $X$ is orderable iff:

(1) $X-Q$ is not a proper compact open subset of $X$.

(2) If $J$ is an open interval of $(T,<)$ and $p \in X-J$ such that $(J \cap(X-$ $Q)) \cup\{p\}$ is compact and the intersection of the closures of $J \cap(X-Q)$ and $J \cap Q$ is $\{p\}$, then the component of $X$ containing $p$ is a singleton.

(3) If $S \notin \mathcal{G}$, then for each member $I$ of $\mathcal{F}$, there is a point $f(I) \in X-I$ such that if $\mathcal{L} \subseteq \mathcal{F}$ and $p \in X-((\cup \mathcal{E}) \cup f(\mathcal{E}))$, then $p$ is in the closure of $X \cap(\cup \mathcal{L})$ iff $p$ is in the closure of $f(\mathcal{E})$.

What will first be shown is that the result obtained from Lemma 4.3 as follows is true: (a) replace in the hypothesis of Lemma 4.3 the condition that $X$ is first countable by the stronger condition that $X$ is metrizable, and (b) eliminate condition (3) of Lemma 4.3. Then it will be shown that for any suborderable space $X$ embedded in an ordered space $(T,<)$ condition $(3)$ of Theorem 4.2 is equivalent to condition (2) of Lemma 4.3. These results will show that a suborderable metrizable space $X$ is orderable iff conditions (2) and (3) of Theorem 4.2 are satisfied. This will prove Theorem 4.2.

4.4. LemMA. Let $X$ be a metrizable space embedded in an ordered space ( $T$, $<)$. Then $X$ is orderable iff:

(1) $X-Q$ is not a proper compact open subset of $X$.

(2) If $J$ is an open interval of $(T,<)$ and $p \in X-J$ such that $(J \cap(X-$ $Q)) \cup\{p\}$ is compact and the intersection of the closures of $J \cap(X-Q)$ and $J \cap Q$ is $\{p\}$, then the component of $X$ containing $p$ is a singleton.

Proof. Let $\left(S, \leqslant^{\prime}\right)$ be an ordered compactification of $T$. To prove this lemma it is sufficient by Lemma 4.3 to show that condition (3) of Lemma 4.3 is satisfied. So let $S \notin \mathcal{G}$.

By Proposition 2.5 there is an admissible convex metric $\partial$ on $X$ bounded by 1. For $I=[p, q] \in \mathcal{F}$ define $\operatorname{diam}(I)=1$ if $(-\infty, p) \cap X=\varnothing$ or $(q$, $\infty) \cap X=\varnothing$. There are at most four members of $\mathscr{F}$ of the form $I=[p, q]$ such that $(-\infty, p) \cap X=\varnothing$ or $(q, \infty) \cap X=\varnothing$ since by the properties of $\mathscr{F}$ given after the statement of Theorem 4.2 if $I_{i}=\left[p_{i}, q_{i}\right]$ is a member of $\mathcal{F}$ for $i=1,2, \ldots, 5$ such that the $I_{i}$ 's are distinct and, for $i=1,2,3,4, q_{i}<{ }^{\prime} p_{i+1}$, then $\left(-\infty, p_{3}\right) \cap X \supseteq I_{1} \cap X \neq \varnothing$ and $\left(q_{3}, \infty\right) \cap X \supseteq I_{5} \cap X \neq \varnothing$. For $I=[p, q] \in \mathscr{F}$ such that $(-\infty, p) \cap X \neq \varnothing$ and $(q, \infty) \cap X \neq \varnothing$ define $\operatorname{diam}(I)=\partial((-\infty, p) \cap X,(q, \infty) \cap X)$. It will now be shown that, for each $I=[p, q] \in \mathscr{F}, \operatorname{diam}(I)>0$. If $\operatorname{diam}(I)=1$, then trivially $\operatorname{diam}(I)>0$. So assume $\operatorname{diam}(I)<1$. Then $(-\infty, p) \cap X \neq \varnothing$ and $(q, \infty) \cap X \neq \varnothing$. By the 
properties of $\mathcal{F}$ given earlier $p \neq q$ and there exists a point $c \in I \cap X$. Then either $p \neq c$ or $q \neq c$. Without loss of generality let $c \neq p$. Thus, $c$ is not a boundary point of $(-\infty, p)$ since $(S,<)$ is ordered and $p<$ ' $c$. Hence, by the convexity of $\partial, \operatorname{diam}(I)>\partial((-\infty, p) \cap X, c)>0$. Thus $\operatorname{diam}(I)>0$.

For $n \in \mathrm{N}$ define $\mathscr{T}_{n}=\{I \in \mathscr{F} \mid 1 /(n+1)<\operatorname{diam}(I)<1 / n\}$. We will define a map $f_{n}: \Re_{n} \rightarrow X$ for each $n \in N$. Then the $f_{n}$ 's define a map $f:$ $\mathscr{F} \rightarrow X$ such that, for each $n \in \mathbf{N}, f \mid \mathscr{T}_{n}=f_{n}$ because $\left\{\mathscr{T}_{n} \mid n \in \mathbf{N}\right\}$ is a decomposition of $\mathcal{F}$. The $f_{n}$ 's will be defined so that $f$ satisfies the properties in condition (3) of Lemma 4.3.

By the properties of $\mathcal{F}$ and $\mathcal{G}$ given after Theorem 4.2, for each $I \in M_{n}$, $(X-I) \neq \varnothing$ since $S \notin \mathcal{G}$. Therefore, since $\partial$ is convex and $\operatorname{diam}(I)<1 / n$, there exists a point $x \in X-I$ such that $\partial(x, I \cap X)<2 / n$. Fix $n \in N$ and well order the members of $M_{n}$ with the least ordinal $\tau$ of the cardinality of $\mathfrak{N}_{n}$, i.e., $\mathfrak{K}_{n}=\left\{I_{\alpha} \mid \alpha<\tau\right\}$. The points in the range of $f_{n}$ will be chosen by induction on $\left\{I_{\alpha} \mid \alpha<\tau\right\}$ such that there are a finite number of points in the range of $f_{n}$ contained in any member of $\Re_{n}$. Choose $f_{n}\left(I_{0}\right)$ to be an element of $X$ outside of $I_{0}$ such that the $\partial$-distance between $f_{n}\left(I_{0}\right)$ and $I_{0} \cap X$ is less than $2 / n$. Such a choice can be made by what was shown in the beginning of this paragraph. If possible, also let $f_{n}\left(I_{0}\right)$ be in a member of $\mathfrak{T}_{n}$. Assume $f_{n}\left(I_{\lambda}\right)$ has been chosen for every $\lambda<\alpha<\tau$. Choose $f_{n}\left(I_{\alpha}\right)$ to be an element of $X$ outside of $I_{\alpha}$ such that the $\partial$-distance between $f_{n}\left(I_{\alpha}\right)$ and $I_{\alpha} \cap X$ is less that $2 / n$ and:

(a) If possible choose $f_{n}\left(I_{\alpha}\right)$ from a member of $\mathfrak{T}_{n}$ which contains no point outside of $I_{\alpha}$ chosen by $f_{n}\left(I_{\lambda}\right)$ for any $\lambda<\alpha$.

(b) If it is not possible to make the choice described in (a), then if possible choose $f_{n}\left(I_{\alpha}\right)$ from a member of $\Re_{n}$ such that $f_{n}\left(I_{\alpha}\right)=f_{n}\left(I_{\lambda}\right)$ for some $\lambda<\alpha$.

(c) If it is not possible to make the choices described in (a) or (b), then if possible simply choose $f_{n}\left(I_{\alpha}\right)$ from a member of $\mathfrak{T}_{n}$. (Note that such a choice may be possible. In fact, if it is not possible to make the choices described in (a) or (b) but $f_{n}\left(I_{\alpha}\right)$ is a point in a member $I$ of $\mathscr{T}_{n}$, then, for some $\lambda<\alpha$, $f_{n}\left(I_{\lambda}\right)$ is a point in $I$ outside of $I_{\alpha}$ such that the $\partial$-distance between $I_{\alpha} \cap X$ and $f_{n}\left(I_{\lambda}\right)$ is not less than $2 / n$. Hence, by the convexity of $\partial, f_{n}\left(I_{\alpha}\right)$ is in the open interval of $(S, \leqslant)$ between $I_{\alpha}$ and $f_{n}\left(I_{\lambda}\right)$ because the $\partial$-distance between $I_{\alpha} \cap X$ and $f_{n}\left(I_{\alpha}\right)$ is less than $2 / n$ and $f_{n}\left(I_{\alpha}\right)$ is not in $I_{\alpha}$.)

(d) If it is not possible to choose $f_{n}\left(I_{\alpha}\right)$ from a member of $\mathfrak{K}_{n}$, then choose $f_{n}\left(I_{\alpha}\right)$ to be a point in the complement of $\cup \mathscr{N}_{n}$ in $S$.

It is not hard to see by inspection that there are at most six (although any finite number would do) points in $f_{n}\left(\mathscr{N}_{n}\right) \cap I$ for each $I \in \mathscr{T}_{n}$. (A formal proof is tedious and detailed but not difficult.)

Since $f_{n}$ has been defined for arbitrary $n \in \mathbf{N}, f$ has been defined where $f_{n}=f \mid \Re_{n}$. 
ClaIM. $f$ satisfies the requirements of condition (3) of Lemma 4.3.

Proof. Let $\mathfrak{E} \subseteq \mathscr{F}$ and let $p \in X-((\cup \mathfrak{L}) \cup f(\mathfrak{L}))$.

A. Let $p \in \overline{X \cap(\cup \mathcal{L})}$. Then there is a monotone sequence $\left\{I_{r}\right\}_{r=1}^{\infty}$ in $\mathfrak{L}$ and for each $r \in \mathbf{N}$ a point $x_{r} \in\left(I_{r} \cap X\right)$ such that $\left\{x_{r}\right\}_{r=1}^{\infty}$ is a monotone sequence converging to $p$. We need to show $\left\{f\left(I_{r}\right)\right\}_{r=1}^{\infty}$ converges to $p$. Hence, it is enough to show that for each $j \in \mathbf{N}$ there exists an $r_{0} \in \mathbf{N}$ such that, for all $r>r_{0}, \partial\left(f\left(I_{r}\right), p\right)<1 / j$. So let $j \in \mathbf{N}$. Choose $r_{0} \in \mathbf{N}$ such that

$$
\partial\left(x_{r_{0}}, p\right)<1 / 4 j \text {. }
$$

Then, by the convexity of $\partial$ for all $r>r_{0}$,

$$
\operatorname{diam}\left(I_{r}\right)<1 / 4 j \text { and } \partial\left(x_{r}, p\right)<1 / 4 j \text {. }
$$

Hence, $\partial\left(f\left(I_{r}\right), I_{r} \cap X\right)<2 / 4 j$. So $\partial\left(f\left(I_{r}\right), x_{r}\right)<3 / 4 j$ and hence, $\partial\left(f\left(I_{r}\right), p\right)$ $<\partial\left(f\left(I_{r}\right), x_{r}\right)+\partial\left(x_{r}, p\right)<1 / j$. Thus, $\left\{f\left(I_{r}\right)\right\}_{r=1}^{\infty}$ converges to $\mid p$. 'So $p \in \overline{f(\mathcal{L})}$.

B. Now let $p \in \overline{f(\mathfrak{L})}$. Then there is a strictly monotone sequence $\left\{p_{r}\right\}_{r=1}^{\infty}$ in $f(\mathfrak{E})$ converging to $p$. First we show by contradiction that $\left\{\operatorname{diam}\left(I_{r}\right)\right\}_{r=1}^{\infty}$ converges to zero where, for each $r \in N, I_{r}$ is any element of $f^{-1}\left(p_{r}\right) \cap$ (UL).

Suppose this is false. Then there exists a sequence $\left\{I_{r}\right\}_{r-1}^{\infty}$ such that $I_{r} \in f^{-1}\left(p_{r}\right) \cap(\cup \mathcal{L})$ for each $r \in \mathbf{N}$ and $\left\{\operatorname{diam}\left(I_{r}\right)\right\}_{r=1}^{\infty}$ does not converge to 0 . Since $\left\{p_{r}\right\}_{r=1}^{\infty}$ is strictly monotone, the members of $\left\{I_{r}\right\}_{r=1}^{\infty}$ are distinct. Hence, there is a subsequence $\left\{I_{r_{1}}\right\}_{i=1}^{\infty}$ of distinct members of $\Re_{n}$ for some fixed $n \in \mathbf{N}$. Now $p \notin \cup\left\{I_{r}, t \in \mathbf{N}\right\}$ because $p \notin \cup \ell$. Thus, $p$ is not an accumulation point of $U\left\{I_{r}, t \in \mathbf{N}\right\}$ since, for each $t \in \mathbf{N}, I_{r_{r}}$ is convex and $1 /(n+1)<\operatorname{diam}\left(I_{r_{r}}\right)$. So there is a $j \in \mathbf{N}$ such that $\partial\left(p_{r}, p\right)<1 /(n+1)$ and no member of $\left\{I_{r_{1}}\right\}_{r=1}^{\infty}$ intersects the open interval between $p_{r_{r}}$ and $p$ because $\left\{p_{r_{1}}\right\}_{t-1}^{\infty}$ converges to $p$. It will be shown that there exists an $i>j+6$ such that $p_{r_{i}} \in \cup \Re_{n}$. Since for every $k \in N$ there is an infinite number of distinct members of $\left\{I_{r_{i}}\right\}_{i=k}^{\infty}$, there is an $i>j+6$ such that at least one member $I_{r_{m}}$ of $\left\{I_{r_{r}}\right\}_{i=1}^{\infty}$ is in the open interval between $p$ and $I_{r_{i}}$. There are no members of $\left\{I_{r_{1}}\right\}_{t=1}^{\infty}$ in the open interval between $p_{r_{1}}$ and $p$ because there are no members of $\left\{I_{r_{1}}\right\}_{i=1}^{\infty}$ in the open interval between $p_{r_{j}}$ and $p$, but $p_{r_{i}}$ is in this open interval. Thus, $I_{r_{m}}$ is in the open interval between $p_{r_{1}}$ and $I_{r_{i}}$. So by the convexity of $\partial$ every point of $I_{r_{m}} \cap X$ is of $\partial$-distance less than $2 / n$ from $I_{r_{i}} \cap X$ because the $\partial$-distance between $p_{r_{i}}=f\left(I_{r_{i}}\right)=f_{n}\left(I_{r_{i}}\right)$ and $I_{r_{1}} \cap X$ is less than $2 / n$. Therefore, by the construction of $f_{n}, p_{r_{1}} \in \cup \Re_{n}$ because $\left(I_{r_{m}} \cap X\right)-I_{r_{1}}$ is not empty by a property of $\mathscr{F}$, and $I_{r_{m}} \cap I_{r_{i}}$ is empty.

Let $K$ be the member of $\mathfrak{N}_{n}$ that contains the point $p_{r_{i}}$. Hence, $p_{r_{j}}$ and $p_{r_{i+6}}$ are not members of $K$ because $i \geqslant j+6$, there are at most 6 points in $f\left(\Re_{n}\right) \cap I=f_{n}\left(\Re_{n}\right) \cap I$ for each $I \in \Re_{n}$, and $\left\{p_{r_{t}}\right\}_{t=1}^{\infty}$ is strictly mono- 
tone. So $K$ is contained in the open interval between $p_{r_{j}}$ and $p_{r_{1+6}}$ since $K$ is convex. Thus, $K$ is contained in the open interval between $p_{r_{j}}$ and $p$ since $p_{r_{1+6}}$ is an element of this open interval. Therefore, $\operatorname{diam}(K)<1 /(n+1)$ because $\partial$ is convex and $\partial\left(p_{r}, p\right)<1 /(n+1)$. However, $1 /(n+1)<\operatorname{diam}(K)$ because $K$ is a member of $\Re_{n}$. This contradiction yields that $\left\{\operatorname{diam}\left(I_{r}\right)\right\}_{r=1}^{\infty}$ converges to 0 .

Finally we can show that $p$ is a cluster point of \{\}$_{r=1}^{\infty}$. So choose $x_{r} \in\left(I_{r} \cap X\right)$ for each $r \in N$ and let $j \in N$. Then there exists an $r_{0} \in \mathbf{N}$ such that, for every $r>r_{0}, \operatorname{diam}\left(I_{r}\right)<1 / 4 j$ and $\partial\left(f\left(I_{r}\right), p\right)<1 / 4 j$. Hence, $\partial\left(f\left(I_{r}\right), I_{r} \cap X\right)<2 / 4 j$. So $\partial\left(f\left(I_{r}\right), x_{r}\right)<3 / 4 j$. Therefore,

$$
\partial\left(x_{r}, p\right)<\partial\left(x_{r}, f\left(I_{r}\right)\right)+\partial\left(f\left(I_{r}\right), p\right)<1 / j .
$$

Hence, $\left\{x_{r}\right\}_{r=1}^{\infty}$ converges to $p$. So $p \in \overline{X \cap(\cup \mathcal{L})}$.

Thus, the claim is proved that $f$ satisfies the requirements of condition (3) of Lemma 4.3. So by Lemma 4.3, this lemma is proved.

4.5. Lemma. Let $X$ be a subspace of an ordered space $(T,<)$. Then condition (3) of Theorem 4.2 is equivalent to condition (2) of Lemma 4.3.

Proof. Let condition (3) of Theorem 4.2 hold. It will be shown by contradiction that condition (2) of Lemma 4.3 holds. So let $J$ be an open interval of $T$ and $p \in(X-J)$ such that $(J \cap(X-Q)) \cup\{p\}$ is compact and the intersection of the closures of $J \cap(X-Q)$ and $J \cap Q$ is $\{p\}$. Suppose the component $K$ of $X$ containing $p$ is nondegenerate. Since $p$ is a boundary point in $X$ of $K, p$ is an endpoint of $K$. Without loss of generality let $p$ be the left endpoint of $K$. So every point of $J$ is less than $p$ because $p$ is a boundary point of the interval $J$, and $K$ is nondegenerate. Let $W=J \cap X$. Then $W$ is a neighborhood of $p$ relative to the complement in $X$ of $K$ since $J$ is an open interval of $T, W^{\prime}=(J \cup K) \cap X=(J \cap X) \cup K$ is a neighborhood of $p$ in $X$, and $W^{\prime}-K=((J \cup K) \cap X)-K=J \cap X=W$. Note that $W-Q=(J \cap X)-Q=J \cap(X-Q)$ and $W \cap Q=(J \cap X) \cap Q$ $=J \cap Q$. So $\{p\}$ is the intersection of the closures of $W-Q$ and $W \cap Q$. Now $(W-Q) \cup\{p\}=(J \cap(X-Q)) \cup\{p\}$ is closed in $X$ because it is compact. Hence, $(W-Q) \cup\{p\}$ is the closure of $W-Q$ since $p$ is a boundary point of $W-Q$. So $W-Q$ has compact closure. Thus, $K$ is a singleton since condition (3) of Theorem 4.2 holds and it has been shown that the hypothesis of this condition is satisfied. This contradicts the supposition that $K$ is nondegenerate. Therefore, it is false that $K$ is nondegenerate, i.e., $K$ is a singleton. So condition (2) of Lemma 4.3 holds.

Conversely, let condition (2) of Lemma 4.3 hold. It will be shown by contradiction that condition (3) of Theorem 4.2 holds. So let $W$ be a neighborhood of $p \in X$ relative to the complement in $X$ of $p$ 's component $K$ such that $W-Q$ has compact closure and $\{p\}$ is the intersection of the 
closures of $W-Q$ and $W \cap Q$. Suppose $K$ is nondegenerate. Since $p$ is a boundary point in $X$ of $K, p$ is an endpoint of $K$. Without loss of generality let $p$ be the left endpoint of $K$. So $p$ is a left limit point of both $\overline{W-\bar{Q}}$ and $\overline{W \cap Q}$, since $K$ is nondegenerate. By definition $W \cup K$ is a neighborhood in $X$ of $p$. Hence there are points $x_{0}, x_{1} \in \overline{W-Q}$ such that $x_{0}<x_{1}<p$, there are no other points of $\overline{W-Q}$ between $x_{0}$ and $x_{1}$, and $\left[x_{0}, p\right) \cap X \subseteq W$ because $p$ is a left limit point of the compact nonconnected set $\overline{W-Q}$ and $p$ has a base of interval neighborhoods of $X$. So $x_{1}$ is not a left limit point in $\overline{W-Q}$, and $x_{1}$ is in the interior of $W$ relative to $X$. In addition, $x_{1}$ is not in the closure of $W \cap Q$ since, otherwise, $x_{1}$ would be in the intersection of the closures of $W-Q$ and $W \cap Q$. Hence, $x_{1}$ is not a left limit point in $X$. Since $T$ is ordered, there exists a point $t \in T$ such that $x_{0}<t<x_{1}$ and $\left(t, x_{1}\right) \cap X$ is empty. Let $J=(t, p)$. Then $J$ is an open interval of $T, p \in(X-J)$, and $J \cap X=\left[x_{1}, p\right) \cap X \subseteq W$. Thus, $J \cap(X-Q)=(J \cap X)-Q \subseteq W-$ $Q$, and $J \cap Q=(J \cap X) \cap Q \subseteq W \cap Q$. So $p$ is the only possible element in both the closures of $J \cap(X-Q)$ and $J \cap Q$. However, $p$ is an element of both the closures of $J \cap(X-Q)$ and $J \cap Q$ because $p$ is the right boundary point of the open interval $J$ and $p$ is a left limit point of both $\overline{W-Q}$ and $\overline{W \cap Q}$, i.e., $p$ is a boundary point of both $(-\infty, p) \cap(W-Q)$ and $(-\infty$, $p) \cap(W \cap Q)$. Thus, $\{p\}$ is the intersection of the closures of $J \cap(X-Q)$ and $J \cap Q$. To show that $(J \cap(X-Q)) \cup\{p\}$ is compact it will first be shown that $p$ is the only point in $\overline{J \cap(X-Q)}-(J \cap(X-Q))$. However, if there were another such point $q$, then $q$ would have to be in $J \cap X$ because $\overline{J \cap(X-Q)} \subseteq \overline{J \cap X}=\overline{\left.x_{1}, p\right) \cap X}=\left[x_{1}, p\right] \cap X=(J \cap X) \cup\{p\} ;$

so $q$ would be a point distinct from $p$ in the intersection of the closures of $J \cap(X-Q)$ and $J \cap Q$. Hence, $\overline{J \cap(X-Q)}=(J \cap(X-Q)) \cup\{p\}$. Therefore, $(J \cap(X-Q)) \cup\{p\}$ is compact since it is an $X$-closed subspace of the compact space $\overline{W-Q}$. Thus, $K$ is a singleton because condition (2) of Lemma 4.3 holds and it has been shown that the hypothesis of this condition has been satisfied. This contradicts the supposition that $K$ is nondegenerate. Therefore, it is false that $K$ is nondegenerate, i.e., $K$ is a singleton. So condition (3) of Theorem 4.2 holds.

Hence, condition (3) of Theorem 4.2 is equivalent to condition (2) of Lemma 4.3.

Proof of Theorem 4.2. Theorem 4.2 now follows directly from Lemmas $4.3,4.4$, and 4.5 .

4.6. Corollary. A suborderable metrizable space $X$ is orderable if it satisfies any of the following:

(a) $X$ is compact.

(b) $X$ is connected. 
(c) The closure of the union of the components of $X$ with no endpoints intersects each component of $X$.

(d) The closure of the union of the singleton components of $X$ intersects each component of $X$.

(e) $X$ is totally disconnected.

(f) Each nondegenerate component of $X$ has a compact neighborhood.

(g) No component of $X$ has a compact neighborhood.

(h) $X$ contains no proper compact open subset.

(i) $X$ contains neither singleton components nor compact open components.

(j) $X$ contains no compact components.

(k) The union of the components of $X$ with no endpoints is not closed, and each nondegenerate component of $X$ is open.

(l) $X$ is locally compact, and the union of all singleton components of $X$ is a proper closed noncompact subset of $X$.

(m) $X$ is locally compact, and there is at least one open nondegenerate component of $X$ with precisely one endpoint.

4.7. COROLlary. A suborderable metrizable space $X$ is orderable if it satisfies any of the following:

(a) $Q$ is not a proper subset of $X$.

(b) $Q$ is clopen, and $X-Q$ is not compact.

(c) $X$ is locally compact, but $X-Q$ is not compact.

(d) $Q$ is not closed, and each nondegenerate component of $X$ is open.

(e) The closure of $Q$ intersects each component of $X$.

(f) $Q$ is dense in $X$.

(g) The closure of $X-Q$ intersects each component of $X$.

(h) $X-Q$ is closed but not compact and is the union of the singleton components of $X$.

Recall the definition of $U$ in the beginning of $\S 3$. It can be checked by examining the definitions on p. 388 in [Ru] that each member of $\mathscr{F}$ contains at least one component of $X$ and, hence, at least one point of $U$. Using this fact it is not hard to verify that if $X$ is occasionally switched to $U$ in the proof of Lemma 4.4, then we obtain a proof of Lemma 4.4 when $U$ is metrizable instead of $X$. Hence we obtain the following counterpart of Corollary 3.5.

4.8. CoROllary. Let $X$ be a space each of whose components has at most two boundary points, and let $U$ be metrizable. Then $X$ is orderable iff conditions (1), (2), and (3) of Theorem 4.2 hold.

\section{REFERENCES}

[C] E. Čech, Topological spaces, 2nd ed., Publ. House Czech Acad. Sci., Prague, 1966; English transl. Wiley, New York, 1966. MR 21 \#2962; 35 \#2254. 
[D-W] J. van Dalen and E. Wattel, $A$ topological characterization of ordered spaces, General Topology and Appl. 3 (1973), 347-354. MR 49 \#6182.

[E] S. Eilenberg, Ordered topological spaces, Amer. J. Math. 63 (1941), 39-45. MR 2, 179.

$\left[\mathbf{H}_{1}\right]$ H. Herrlich, Ordnungsfähigkeit topologischer Räume, Inaugural-Dissertation der Freien Universität Berlin, Berlin, 1962.

$\left[\mathrm{H}_{2}\right] \_$, Ordnungsfähigkeit total-diskontinuierlicher Räume, Math. Ann. 159 (1965), 77-80. MR 32 \#426.

$\left[\mathrm{H}_{3}\right]$, Ordnungsfähigkeit zusammenhängender Räume, Fund. Math. 57 (1965), 305-311. MR 32 \#3028.

$\left[\mathrm{H}_{4}\right]-$, Mengen reeler Zahlen. Ordnungstheoretische und topologische Kennzeichnung, Prace Mat. 11 (1968), 205-212. MR 37 \#2185.

[ $\left.\mathrm{H}_{3}\right]$, Problems, Proc. Internat. Sympos. Topology and Its Applications (HercegNovi, 25-31. 8. 1968, Yugoslavia), Savez Društava Mat. Fiz. Astron., Belgrade, 1969, p. 345.

[Kok] H. Kok, On conditions equivalent to the orderability of a connected space, Nieuw Arch. Wisk. (3) 18 (1970), 250-270. MR 45 \#9295.

[Kow] H.-J. Kowalsky, Topologische Räume, Birkhäuser Verlag, Basel, 1961. MR 22 \# 12502.

[L] D. J. Lutzer, On generalized ordered spaces, Dissertationes Math. Rozprawy Mat. 89 (1971), 32 pp. MR 48 \#3018.

$\left[\mathrm{P}_{1}\right] \mathrm{S}$. Purisch, The orderability and suborderability of topological spaces, $\mathrm{Ph}$. D. Thesis, Carnegie-Mellon University, 1973.

$\left[\mathbf{P}_{2}\right]-$ On the orderability of Stone-Cech compactifications, Proc. Amer. Math. Soc. 41 (1973), 55-56. MR 48 \# 5005.

$\left[\mathbf{P}_{3}\right]$, Projectives in the category of ordered spaces, Studies in Topology (Proc. Conf., Univ. North Carolina, Charlotte, N. C., 1974), Academic Press, New York, 1975, pp. 467-478. MR 50 \# 11187.

[Ro] Prabir Roy, Failure of equivalence of dimension concepts for metric spaces, Bull. Amer. Math. Soc. 68 (1962), 609-613. MR 25 \#5495.

[Ru] M. E. Rudin, Interval topology in subsets of totally orderable spaces, Trans. Amer. Math. Soc. 118 (1965), 376-389. MR 31 \#3994.

Department of Mathematics, University of Ibadan, Ibadan, Nigeria 